\title{
Practice Changes to Improve Delivery of Surgical Antibiotic Prophylaxis
}

Rosemary Zvonar, Pam Bush and Virginia Roth

\begin{abstract}
Timely administration of appropriate antibiotics preoperatively can decrease the incidence of surgical site infection. We evaluated compliance with quality indicators in the delivery of antimicrobial surgical prophylaxis at The Ottawa Hospital and assessed the impact of a change to the hospital's Surgical Prophylaxis Policy.

An audit in 2002 revealed improvement was necessary in the timing of preoperative doses, dosing for patients with a high body mass index, and intra-operative redosing. As a result, a multidisciplinary group was formed and a new surgical prophylaxis policy was approved. The policy included administration of preoperative doses by the anesthesiologist, and an automatic substitution for higher doses of antibiotics for select patients. This practice change resulted in significant improvements to the preoperative timing and dosing in subsequent audits. A mechanism to address intra-operative redosing will be implemented.
\end{abstract}

\section{Background and Objectives}

Surgical site infections (SSIs) are associated with increased patient morbidity, mortality and healthcare costs (Kirkland et al. 1999). It has been well established that properly timed preop- erative antibiotics at the correct dose reduce the incidence of SSIs for a variety of surgical procedures (Classen et al. 1992; Forse et al. 1989; van Kasteren et al. 2007). Additional aspects of surgical prophylaxis - such as selection of the appropriate agent(s), administration of intra-operative doses and duration of post-operative prophylaxis - are also important components of antimicrobial stewardship and infection prevention. Patient safety agencies in both the United States and Canada have identified the prevention of SSIs and appropriate antimicrobial prophylaxis as a primary quality target.

The objectives of this study were to evaluate compliance with quality indicators in the delivery of antimicrobial surgical prophylaxis at The Ottawa Hospital and to assess the impact of a change to the hospital's Surgical Prophylaxis Policy.

\section{Methods}

Quality indicators for antibiotic surgical prophylaxis were derived from the current literature (American Society of Health-System Pharmacists 1999; "Antimicrobial Prophylaxis in Surgery" 2001; Auerbach 2001; Dellinger et al. 1994; Forse et al. 1989; Mangram et al. 1999; Scottish Intercollegiate Guidelines Network 2000; Swoboda et al. 1996; Zanetti et al. 2001). A baseline audit was performed between January and March 2002 to assess compliance with the quality indicators in five surgical divisions (Orthopedic, Neurosurgery, Vascular, Thoracic and General Surgery). As part of the continuous quality improvement process, the antibiotic prophylaxis quality 
Table 1. Compliance with surgical prophylaxis quality indicators

\begin{tabular}{|l|l|l|l|l|l|}
\hline Date of Audit & $\begin{array}{l}\text { Total Charts } \\
\text { Reviewed* }\end{array}$ & $\begin{array}{l}\text { Appropriate } \\
\text { Agent }\end{array}$ & $\begin{array}{l}\text { OR (95\% CI); } \\
\text { p Valuet }\end{array}$ & $\begin{array}{l}\text { Appropriate } \\
\text { Dose }\end{array}$ & $\begin{array}{l}\text { OR (95\% CI); } \\
\text { p Valuet }\end{array}$ \\
\hline Jan-Mar 2002 & 290 & $241 / 258,93.4 \%$ & - & $173 / 239,72.4 \%$ & - \\
\hline May-July 2004 & 261 & $217 / 235,92.3 \%$ & $0.8(0.4-1.8) ; .64$ & $180 / 217,83 \%$ & $1.9(1.2-3.0) ; .007$ \\
\hline June-Sept 2006 & 273 & $234 / 253,92.5 \%$ & $1.0(0.5-2.1) ; .9$ & $208 / 232,89.7 \%$ & $1.8(0.9-3.2) ; .04$ \\
\hline
\end{tabular}

\begin{tabular}{|l|l|l|l|l|l|l|}
\hline Date of Audit & $\begin{array}{l}\text { Appropriate } \\
\text { Administration }\end{array}$ & $\begin{array}{l}\text { OR (95\% CI); } \\
\text { p Valuet }\end{array}$ & $\begin{array}{l}\text { Intra-operative } \\
\text { Dose Given if } \\
\text { Required }\end{array}$ & $\begin{array}{l}\text { OR (95\% CI); } \\
\mathbf{p} \text { Valuet }\end{array}$ & $\begin{array}{l}\text { Post-operative } \\
\text { Duration } \leq 24 \text { Hours }\end{array}$ & $\begin{array}{l}\text { OR (95\% CI); } \\
\text { p Valuet }\end{array}$ \\
\hline Jan-Mar 2002 & $94 / 261,36 \%$ & - & $13 / 33,39.4 \%$ & - & $233 / 260,89.6 \%$ & - \\
\hline May-July 2004 & $170 / 251,67.7 \%$ & $3.7(2.5-5.5) ;<.001$ & $27 / 50,54 \%$ & $1.8(0.7-0.9)_{;} .19$ & N/A & - \\
\hline June-Sept 2006 & $205 / 261,78.5 \%$ & $1.7(1.2-2.6) ; .006$ & $22 / 58,37.9 \%$ & $0.5(0.2-1.2) ; .09$ & $236 / 264,89.4 \%$ & $\begin{array}{l}1.0 \\
(0.5-1.8) ; .9\end{array}$ \\
\hline
\end{tabular}

$\mathrm{Cl}=$ confidence interval; $\mathrm{N} / \mathrm{A}=$ not assessed; $\mathrm{OR}=$ odds ratio.

${ }^{*}$ Note: Denominators to do not equal total charts reviewed due to missing data.

tCompared with the previous audit period.

$\ddagger$ As the duration of post-operative prophylaxis did not exceed 24 hours in $90 \%$ of the cases in the 2002 audit, it was not re-examined in 2004 .

indicators were prospectively re-evaluated between May and July 2004 and between June and September 2006. For each audit, a convenience sample of approximately 50 patient charts per division were reviewed.

\section{Findings and Solutions}

A summary of the audit results is shown in Table 1 . The baseline audit revealed that the selection of antimicrobial agents and duration of post-operative prophylaxis were appropriate in $90 \%$ of cases. However, only $40 \%$ of patients received an intraoperative dose of antibiotic when indicated. In addition, only one third of patients received a preoperative dose of antibiotic within the recommended time period. Finally, only $15 \%$ (seven of 47) of patients with a large body mass index (BMI) received an antibiotic dose that was greater than the standard recommended dose.

Following the baseline audit, a multidisciplinary team was formed to review the institution's process for antimicrobial prophylaxis and to make recommendations for improvement. Members included the antimicrobial pharmacist, the clinical director, clinical managers and nurse educators for peri-operative services, the pharmacy operations manager and physician representatives from surgery, anesthesiology and infection prevention and control. The working group recommended that the preoperative antibiotics be administered by the anesthesiologist at the induction of anesthesia (with the exceptions of vancomycin and clindamycin due to their longer administration times). Orders written as "preop" or "on call to OR" were to be understood as indicating that the antibiotic was to be administered in the operating room (OR) as per this new protocol. To facilitate delivery, premixed preoperative doses of antibiotics were to be sent to the OR attached to a patient's chart and were also made readily available in the OR suites. These interventions, along with additional practice changes to facilitate the process (Table 2), were implemented in June 2003.

The subsequent audit, conducted in 2004, demonstrated that preoperative antibiotics were four times more likely to be administered in a timely manner and that the dosing was two times more likely to be appropriate following these interventions (see Table 1). These increases were statistically significant. In addition, an intra-operative dose was given when required to a higher percentage of patients, although this increase was not significant. In this audit, a higher antibiotic dose was administered in $63 \%$ ( 44 of 70 ) of patients with a BMI $\geq 30$ or weight $\geq 90$ kilograms. Selection of the appropriate agent occurred in 92.3\% of instances, consistent with the 2002 audit.

Despite these improvements, our desired target of $90 \%$ for all quality indicators was not yet attained. Therefore, a preoperative pause was implemented in March 2005 during which verification is made of the patient, the procedure, the surgical site and whether the preoperative antibiotic has been administered prior to the initial incision. The subsequent audit in 2006 demonstrated a further statistically significant improvement in the percentage of patients who received a preoperative dose of antibiotic within the recommended time period $(78.5 \%$, versus $67.7 \%$ in 2004). There was also a non-statistically significant 
Table 2. Strategies implemented to improve delivery of surgical antibiotic prophylaxis at The Ottawa Hospital

1. Implementation of a new Surgical Prophylaxis Policy (June 2003):

- Anesthesiologist to administer preoperative antibiotic(s) at induction of anesthesia

- Patients with a body mass index (BMI) $\geq 30$ (or weight $\geq 90 \mathrm{~kg}$ if BMI not available) to receive a $2 \mathrm{~g}$ dose of cefazolin, or a 1,500 mg dose of vancomycin

- Surgeon to confirm that any required prophylactic antibiotic(s) have been administered

- Antibiotics to be redosed intra-operatively for surgeries $>3-4$ hours (6 hours for vancomycin) or for significant blood loss $(\geq 1,500 \mathrm{~mL})$

2. Nursing-/pharmacy-initiated automatic substitution to higher doses of antibiotics for patients with a BMI $\geq 30$ or weight $\geq 90 \mathrm{~kg}$

3. Antibiotic prophylaxis added to preoperative checklist

4. Development of a list of surgeries requiring prophylaxis and recommended agents

5. Provision of premixed antimicrobial agents and improved availability in the operating room and pre-admission units

6. Education regarding cross-allergy with cephalosporins in patients with penicillin allergy

7. Implementation of a preoperative pause (March 2005)

increase in the percentage of patients administered an appropriate dose due to a greater proportion of patients $(72 \%$ or 64 of 89) with a high BMI receiving the recommended antibiotic dose. Target levels were maintained for the selection of agent and for post-operative duration of prophylaxis, but the initial improvement in the number of patients who received an intraoperative dose of antibiotic when required returned to the baseline level of the initial review in 2002.

In the subset of patients administered the prophylactic dose of antibiotic prior to incision, the median time between administration and incision decreased from 59 minutes in 2002 to 25 minutes in both 2004 and 2006.

\section{Discussion}

The development and therefore prevention of SSIs involves a number of factors such as skin preparation, surgical technique and specific patient factors (Dellinger et al. 2005; Sessler and Akça 2002). Although surgical antibiotic prophylaxis is but one of these, giving the appropriate antibiotic at the appropriate dose within one hour of incision has been well documented to reduce the incidence of SSIs (Classen et al. 1992; Forse et al. 1989; van Kasteren et al. 2007) and should be expected by all patients undergoing surgeries in which prophylaxis is recommended. Conversely, an unnecessarily prolonged duration of prophylaxis increases the risk of antimicrobial-resistant organisms and adverse events (Harbarth et al. 2000; Kreisel et al. 1995).

Our baseline audit demonstrated that appropriate antimicrobial agents were administered and prophylaxis was correctly limited to 24 hours or less in $90 \%$ of the procedures we reviewed, and remained consistent throughout the study period. However, we found important deficiencies in the timing of the initial preoperative dose, as well as intra-operative redosing - an indicator not frequently evaluated in studies assessing surgical prophylaxis. Other studies have also demonstrated that surgical prophylaxis continues to be suboptimally administered in 25$50 \%$ of cases (Auerbach 2001; Matuschka et al. 1997; Silver et al. 1996; Webb et al. 2006). For example, in a review of over 34,000 surgical procedures in 2,965 acute care US hospitals, the appropriate antimicrobial agent was selected in $92.6 \%$ of procedures; however, timing was appropriate in only $55.7 \%$ of procedures, and only $40.7 \%$ of procedures had antibiotics discontinued within 24 hours of surgery (Bratzler et al. 2005).

Our quality improvement efforts focused on improving the timing, intra-operative administration and dosing of antibiotic prophylaxis. Having the anesthesiologist administer the antibiotic at the time of induction of anesthesia resulted in a significant improvement in the timing of the preoperative dose of prophylactic antibiotic (initially appropriate in $36 \%$ and improving to $68 \%)$ and a decrease in the median interval between antibiotic administration and incision (from 59 to 25 minutes). Although there may be some reluctance on the part of the anesthesiologists to assume this task, this method has also been shown to improve antibiotic administration in other studies too (Matuschka et al. 1997; Webb et al. 2006; White and Schneider 2007). We demonstrated a further increase in the number of patients receiving the antibiotics within the designated time following the introduction of the preoperative pause.

In addition, we observed a significant increase over time in the percentage of patients who received an appropriate dose of preoperative antibiotic. Administration of higher doses to patients with a larger BMI accounted for the majority of this improvement and was most likely related to increased awareness and our automatic substitution policy.

Our quality improvement efforts did not have a sustained impact on the percentage of patients who received intra-operative doses of antibiotics for long procedures or excessive blood loss, although the study was underpowered to detect small changes. One of the most important barriers was that no group (surgeons, anesthesiologists or nursing) was willing to take responsibility for monitoring this parameter. Clearly, a formal process, a mechanism and assignment of responsibility are required to improve this important aspect of surgical prophylaxis. 
This study was not designed to measure the impact of improved antibiotic delivery on the risk of SSIs. A crude analysis of SSI rates during this time period suggested a non-sustained decrease in vascular surgery infection rates following these improvement initiatives. There was no discernible decrease in infection rates in other surgical procedures for which surveillance data were available. The causes of SSIs are usually multi-factorial, and reducing infection rates typically requires multi-faceted interventions. Nonetheless, a decrease in SSIs following an improvement in the timeliness of antibiotic administration has been observed in a number of recent studies (Dellinger et al. 2005; van Kasteren et al. 2007; Webb et al. 2006).

In summary, optimizing delivery of surgical antibiotic prophylaxis is an important patient safety goal. These findings demonstrate that significant improvements in the dosing and timing of preoperative antibiotic prophylaxis can be achieved through continuing quality improvement efforts involving multidisciplinary collaboration. HQ

\section{About the Authors \\ Rosemary K. Zvonar, BScPhm, ACPR, is an antimicrobial pharmacy specialist with the Department of Pharmacy, The Ottawa Hospital, Ottawa, Ontario. You can contact her at 613- 798-5555, ext. 15063, by fax at 613-761-4350 or by e-mail at rzvonar@ottawahospital.on.ca.}

Pam Bush, BScN, RN, is clinical director, Perioperative Services, The Ottawa Hospital.

Virginia Roth, MD, FRCPC, is a hospital epidemiologist and director of the Infection Prevention and Control Program, The Ottawa Hospital.

\section{References}

American Society of Health-System Pharmacists. 1999. "ASHP Therapeutic Guidelines on Antimicrobial Prophylaxis in Surgery." American Journal of Health-System Pharmacy 56: 1839-88.

"Antimicrobial Prophylaxis in Surgery." 2001. Medical Letter 43: 92-7.

Auerbach, A.D. 2001. "Prevention of Surgical Site Infections." In K.G. Shojania, B.W. Duncan, K.M. McDonald and R.M. Wachter, eds., Making Health Care Safer: A Critical Analysis of Patient Safety Practices (Evidence Report/Technology Assessment: No. 43. AHRQ Publication No. 01-E058). Rockville, MD: Agency for Healthcare Research and Quality.

Bratzler, D.W., P.M. Houck, C. Richards, L. Steele, E.P. Dellinger, D.E. Fry, P.M.C. Wright, A. Ma, K. Carr and L. Red. 2005. "Use of Antimicrobial Prophylaxis for Major Surgery: Baseline Results from the National Surgical Infection Prevention Project." Archives of Surgery 140(2): 174-82.

Classen, D.C., R.S. Evans, S.L. Pestotnik, S.D. Horn, R.L. Menlove and J.P. Burke. 1992. "The Timing of Prophylactic Administration of Antibiotics and the Risk of Surgical-Wound Infection." New England Journal of Medicine 326: 281-6.

Dellinger, E.P., P.A. Gross, T.L. Barrett, P.J. Krause, W.J. Martone, J.E. McGowan Jr., R.L. Sweet and R.P. Wenzel. 1994. "Quality Standard for
Antimicrobial Prophylaxis in Surgical Procedures." Clinical Infectious Diseases 18: 422-7.

Dellinger, E.P., S.M. Hausmann, D.W. Bratzler, R.M. Johnson, D.M. Danial, K.M. Bunt, G.A. Baumgardner and J.R. Sugarman. 2005. "Hospitals Collaborate to Decrease Surgical Site Infections." American Journal of Surgery 190(1): 9-15.

Forse, R.A., B. Karam, L.D. MacLean and N.V. Christou. 1989. "Antibiotic Prophylaxis for Surgery in Morbidly Obese Patients." Surgery 106: 750-7.

Harbarth, S., M.H. Samore, D. Lichtenberg and Y. Carmeli. 2000. "Prolonged Antibiotic Prophylaxis after Cardiovascular Surgery and Its Effect on Surgical Site Infections and Antimicrobial Resistance." Circulation 101(25): 2916-21.

Kirkland, K.B., J.P. Briggs, S.L. Trivette, W.E. Wilkinson and D.J. Sexton. 1999. "The Impact of Surgical-Site Infections in the 1990s: Attributable Mortality, Excess Length of Hospitalization, and Extra Costs." Infection Control and Hospital Epidemiology 20: 725-30.

Kreisel, D., T.G. Savel, A.L. Silver and J.D. Cunningham. 1995. "Surgical Antibiotic Prophylaxis and Clostridium difficile Toxin Positivity." Archives of Surgery 130: 989-93.

Mangram, A.J., T.C. Horan, M.L. Pearson, L.C. Silver and W.R. Jarvis. 1999. "Guideline for Prevention of Surgical Site Infection." Infection Control and Hospital Epidemiology 20: 247-78.

Matuschka, P.R., W.G. Cheadle, J.D. Burke and R.N. Garrison. 1997. "A New Standard of Care: Administration of Preoperative Antibiotics in the Operating Room." American Surgeon 63(6): 500-3.

Scottish Intercollegiate Guidelines Network. 2000. Antibiotic Prophylaxis in Surgery (SIGN Publication No. 45). Edinburgh, Scotland: Author. Retrieved August 10, 2007. <http://www.sign.ac.uk/ guidelines/fulltext/45/index.html>.

Sessler, D.I. and O. Akça. 2002. "Nonpharmacological Prevention of Surgical Wound Infections." Clinical Infectious Diseases 35: 1397404.

Silver, A., A. Eichorn, J. Kral, G. Pickett, P. Barie, V. Pryor and M. Dearie. 1996. "Timeliness and Use of Antibiotic Prophylaxis in Selected Inpatient Surgical Procedures." American Journal of Surgery 171: 548-52.

Swoboda, S.M., C. Merz, J. Kostuik, B. Trentler and P.A. Lipsett. 1996. "Does Intraoperative Blood Loss Affect Antibiotic Serum and Tissue Concentration?" Archives of Surgery 131: 1165-72.

van Kasteren, M.E.E., J. Manniën, A. Ott, B-J. Kullberg, A.S. de Boer and I.C. Gyssens. 2007. "Antibiotic Prophylaxis and the Risk of Surgical Site Infections following Total Hip Arthroplasty: Timely Administration Is the Most Important Factor." Clinical Infectious Diseases 44(1): 921-7.

Webb, A.L.B., R.L. Flagg and A.S. Fink. 2006. "Reducing Surgical Site Infections through a Multidisciplinary Computerized Process for Preoperative Prophylactic Antibiotic Administration.” American Journal of Surgery 192: 663-8.

White, A. and T. Schneider. 2007. "Improving Compliance with Prophylactic Antibiotic Administration Guidelines." AORN Journal 85(1): 173-80.

Zanetti, G., R. Giardina and R. Platt. 2001. "Intraoperative Redosing of Cefazolin and Risk for Surgical Site Infection in Cardiac Surgery." Emerging Infectious Diseases 7(5): 828-31. 